\title{
Role of Magnetic Resonance Imaging in Evaluation of the Brain Lesions in HIV-infected Patients: A Prospective Observational Study
}

\author{
Rohan Bhanushali ${ }^{1}$, Pradeepgoud H. Patil ${ }^{2}$ \\ ${ }^{1} \mathrm{MD}$ Student, Department of Radio-Diagnosis, Jawaharlal Nehru Medical College, Belagavi-590010, Karnataka, ${ }^{2}$ Professor, \\ Department of Radio-Diagnosis, Jawaharlal Nehru Medical College, Belagavi-590010, Karnataka, India.
}

Corresponding author: Dr. Pradeepgoud H. Patil, Department of Radio-diagnosis, Jawaharlal Nehru Medical College, Nehru Nagar KLE University, Belagavi 590010, Karnataka, India

DOI: http://dx.doi.org/10.21276/ijcmsr.2019.4.3.7

How to cite this article: Rohan Bhanushali, Pradeepgoud H. Patil. Role of magnetic resonance imaging in evaluation of the brain lesions in HIV-infected patients: a prospective observational study. International Journal of Contemporary Medicine Surgery and Radiology. 2019;4(3):C34-C37.

\section{A B S T R A C T}

Introduction: Human immunodeficiency virus (HIV) infection of the central nervous system may lead to various neurological complications including opportunistic infections of the brain and neurocognitive disorders. Characterization and differentiation of the pathological changes in the central nervous system (CNS) may help in the right diagnosis and treatment.

Material and Methods: The study included 34 patients diagnosed as HIV-positive by enzyme-linked immunosorbent assay. Brain magnetic resonance imaging (MRI) of all the patients was carried out using 1.5 Tesla Symphony Maestro class-MRI. The test was performed using field-of-view of $230 \mathrm{~mm}$, slice thickness of $4 \mathrm{~mm}$ and a matrix size of $256 \times 256$. Spin-echo T1 and T2-weighted (axial/sagittal), fluid-attenuated inversion recovery (axial), diffusion-weighted imaging, and gadoliniumenhanced fat-suppressed T1-weighted sequences were obtained and analyzed.

Results: Toxoplasmosis was the most prevalent diagnosis observed (26.5\%). The majority of the lesions were observed in both supratentorial and infratentorial regions (58.8\%). T1-weighted MRI showed more hypointense lesions in $70.6 \%$ of patients, whereas T2-weighted MRI showed more hyperintensive lesions (79.4\%). Diffusion restriction was present in cases of tubercular etiology (11.7\%), toxoplasmosis (8.8\%) and lymphoma (8.8\%).

Conclusion: MRI can be used to differentiate and characterize various brain lesions in HIV-infected patients. Its high sensitivity in identifying lesions benefits to understand the pathological changes and disease progression in HIV-infected patients.

Keywords: Magnetic Resonance Imaging, Neurologic Manifestations, HIV Infection, Acquired Immunodeficiency Syndrome, Toxoplasmosis

\section{INTRODUCTION}

Infection by the human immunodeficiency virus (HIV) can lead to diverse clinical symptoms. As HIV comes under lentivirus category, they are capable of producing chronic neurologic damage to its animal host. Transmigration of HIV-infected CD4+ cells across the blood-brain barrier causes the infection to the resident cells of central nervous system (CNS) ${ }^{1,2}$

HIV-associated CNS abnormalities can be mainly in the form of lesions, infections, and neoplasms. It may represent as neurocognitive disorders, neuropathy, lymphomas, dementia, vacuolar myelopathy, psychological conditions, and neurological opportunistic infections. Opportunistic infections are one of the major causes that exacerbate the condition of HIV-infected patients with high level of morbidity and mortality rate. These include leukoencephalopathy, toxoplasmosis, cryptococcal meningitis, tuberculosis, neurosyphilis etc. ${ }^{3,4}$
Imaging technologies play an important role in guiding accurate diagnosis and proper therapy in neuronal complications induced by HIV. Impact of HIV in the CNS can be well assessed by magnetic resonance imaging (MRI). MRI can favorably show the range and depth of soft tissue infections, and necrosis and has a higher sensitivity to pick up new lesions; hence making it the modality of choice. It helps to understand the pathological process and disease progression, non-invasively. ${ }^{5,6}$ Therefore, in the present study, MRI was used to differentiate and characterize the brain lesions in the HIV-infected patients.

\section{MATERIAL AND METHODS}

This prospective observational study was conducted at the Department of Radio-Diagnosis, KLE'S Dr. Prabhakar Kore Hospital and Medical Research Centre, Belgaum from January 2015 to December 2015. Ethical clearance was obtained from the institutional ethics committee, Jawaharlal Nehru Medical College, Belgaum, before the commencement 
of the study.

\section{Participants}

The study included 34 patients diagnosed as HIV-positive by enzyme-linked immunosorbent assay referred to the Department of Radio-Diagnosis for brain MRI. Patients those fulfilled the selection criteria were informed about the nature and purpose of the study and were enrolled after obtaining a written informed consent. Patients with ferromagnetic implants, pacemakers and aneurysm clips and those without any visualized MRI pathology were excluded from the study.

\section{Imaging procedure}

Brain MRI of all patients was carried out using 1.5 Tesla Symphony Maestro class-MRI with the help of a dedicated brain coil. The test was performed using a field of view of $230-\mathrm{mm}$, slice thickness of $4 \mathrm{~mm}$ and matrix size of $256 \times 256$ $\mathrm{mm}$. Spin-echo T1 and T2-weighted (axial/sagittal), fluidattenuated inversion recovery (axial), diffusion-weighted imaging (DWI), and gadolinium-enhanced fat-suppressed T1-weighted sequences were obtained. Susceptibilityweighted imaging and magnetic resonance spectroscopy sequences of the brain were obtained in required cases. The collected data were expressed as proportions and percentages as per the suitability.

\section{RESULTS}

A total of $34 \mathrm{HIV}$-positive patients (25 men and 09 women) were studied with various neuronal lesions. Diagnosis and frequency distribution of lesions are shown in Table 1. Toxoplasmosis was the most prevalent (26.5\%) diagnosis observed in the study population. Lesions due to encephalitis and tubercular etiology were observed in $20.6 \%$ and $17.6 \%$ of patients, respectively. Progressive multifocal

\begin{tabular}{|c|c|c|c|c|c|c|}
\hline Lesions & Toxoplasmosis & Tubercular & Encephalitis & PML & Lymphoma & Cryptococcal \\
\hline Frequency, $(n, \%)$ & 9 (26.5) & $7(20.6)$ & $6(17.6)$ & $5(14.7)$ & $4(11.7)$ & $3(8.8)$ \\
\hline \multicolumn{7}{|c|}{ Distribution by location } \\
\hline Supratentorial & $2(5.9)$ & $2(5.9)$ & $1(2.9)$ & $2(5.9)$ & $3(8.8)$ & $3(8.8)$ \\
\hline Infratentorial & - & $1(2.9)$ & - & - & - & - \\
\hline Both & $7(20.6)$ & $4(11.7)$ & $5(14.7)$ & $3(8.8)$ & $1(2.9)$ & - \\
\hline \multicolumn{7}{|c|}{ T1-Weighted intensity of the lesion } \\
\hline Hyperintense & - & - & - & - & - & - \\
\hline Hypointense & $8(23.5)$ & $1(2.9)$ & $6(17.6)$ & $5(14.7)$ & $1(2.9)$ & $3(8.8)$ \\
\hline Isointense & $1(2.9)$ & $6(17.6)$ & - & - & $3(8.8)$ & - \\
\hline Mixed Intense & - & - & - & - & - & - \\
\hline \multicolumn{7}{|c|}{ T2-Weighted intensity of the lesion } \\
\hline Hyperintense & $9(26.5)$ & $4(11.7)$ & $6(17.6)$ & $5(14.7)$ & - & $3(8.8)$ \\
\hline Hypointense & - & $2(5.9)$ & - & - & - & - \\
\hline Isointense & - & - & - & - & $3(8.8)$ & - \\
\hline Mixed intense & - & $1(2.9)$ & - & - & $1(2.9)$ & - \\
\hline
\end{tabular}

\begin{tabular}{|l|c|c|c|c|c|c|}
\hline \multirow{2}{*}{ Diagnosis } & \multicolumn{5}{|c|}{ Type of enhancement } \\
\cline { 2 - 7 } & No enhancement & Ring & Homogeneous & Heterogeneous & Patchy & Nodular \\
\hline Toxoplasmosis & - & $7(20.6)$ & - & - & $1(2.9)$ & $1(2.9)$ \\
\hline Tubercular & - & $6(17.6)$ & - & - & - & $1(2.9)$ \\
\hline Encephalitis & $6(17.6)$ & - & - & - & - & - \\
\hline PML & $5(14.7)$ & - & - & - & - & - \\
\hline Lymphoma & - & - & $3(8.8)$ & $1(2.9)$ & - & - \\
\hline Cryptococcal & $2(5.9)$ & - & $1(2.9)$ & - & - & - \\
\hline PML; Progressive multifocal leukoencephalopathy \\
\hline \multicolumn{7}{|l}{} \\
\hline
\end{tabular}

\begin{tabular}{|l|c|c|c|}
\hline Diagnosis & No meningeal enhancement & Leptomeningeal enhancement & Pachy-meningeal enhancement \\
\hline Toxoplasmosis & $8(23.5)$ & $1(2.9)$ & - \\
\hline Tubercular & $4(11.7)$ & $3(8.8)$ & - \\
\hline Encephalitis & $4(11.7)$ & $1(2.9)$ & - \\
\hline PML & $5(14.7)$ & - & - \\
\hline Lymphoma & $3(8.8)$ & $1(2.9)$ & - \\
\hline Cryptococcal & $2(5.9)$ & $1(2.9)$ & \\
\hline PML; Progressive multifocal leukoencephalopathy & \\
\hline \multicolumn{2}{|r|}{ Table-3: Distribution based on meningeal enhancement pattern on contrast T1-FATSAT sequence (N=34) } \\
\hline
\end{tabular}




\begin{tabular}{|l|c|c|}
\hline Diagnosis & No restriction & Diffusion restriction \\
\hline Toxoplasmosis & $6(17.6)$ & $3(8.8)$ \\
\hline Tubercular & $3(8.8)$ & $4(11.7)$ \\
\hline Encephalitis & $6(17.6)$ & - \\
\hline PML & $5(14.7)$ & - \\
\hline Lymphoma & $1(2.9)$ & $3(8.8)$ \\
\hline Cryptococcal & $3(8.8)$ & - \\
\hline PML; Progressive multifocal leukoencephalopathy \\
\hline \multicolumn{2}{|c|}{ Table-4: Distribution based on diffusion restriction on diffu- } \\
\multicolumn{2}{|c|}{ sion-weighted imaging sequence (N=34) } \\
\hline
\end{tabular}

leukoencephalopathy (PML) was observed in $14.7 \%$ patients, whereas $11.7 \%$ were diagnosed with lymphoma. Lesions due to cryptococcal etiology were the least prevalent diagnosis (8.8\%). Most of our patients were under the age group of $31-40$ years $(38.2 \%)$. In the total study population, altered mental status (94.1\%) was the most prevalent CNS symptom, followed by cognitive impairment (91.1\%), functional impairment (85.3\%), confusion (82.3\%), headache (79.3\%), and behavioral abnormalities (70.6\%).

The majority of the patients was having lesions in both supratentorial and infratentorial regions (58.8\%), whereas $38.23 \%$ of patients having lesions only in the supratentorial region. Results showed that $70.6 \%$ of patients had hypointense lesions and $29.4 \%$ of patients had isointense lesions, as shown by T1-weighted MRI. In this study, $79.4 \%$ of patients had hyperintense lesions, followed by isointense (8.8\%), hypointense (5.9\%), and mixed intense lesions, as observed on T2-weighted MRI. Distribution of patients based on the enhancement pattern of the lesion on contrast T1-FATSAT sequence is represented in Table 2. In the enhancement pattern of the lesion on contrast T1FATSAT (fat saturated) sequence, ring enhancement was observed in $38.2 \%$ of patients. A total of $11.8 \%$ of patients showed homogeneous enhancement, 5.9\% patients showed nodular enhancement, and $2.9 \%$ patients showed patchy and heterogeneous enhancement.

Distribution of patients based on meningeal enhancement pattern on contrast T1-FATSAT sequence is shown in Table 3. Results showed leptomeningeal enhancement in $20.6 \%$ of the patients and $5.9 \%$ with patchy meningeal enhancement. There was no meningeal enhancement in remaining $76.5 \%$ of patients. Most of the leptomeningeal enhancement was due to tubercular etiology. Patchy meningeal enhancements were observed only in the case of encephalitis due to HIV infection. There was no meningeal enhancement in patients with PML. Distribution of patients based on diffusion restriction on DWI sequence is shown in Table 4. Analysis of diffusion restriction on DWI sequence showed a diffusion restriction in $29.4 \%$ of patients. Diffusion restriction was more in cases of tubercular etiology, followed by toxoplasmosis, and lymphoma. There was no diffusion restriction in cases of encephalitis and PML and cryptococcal etiology.

\section{DISCUSSION}

In the present study, various pathological lesions were observed in the CNS of HIV-positive patients. Among those, toxoplasmosis (26.5\%) was the most prevalent diagnosis observed. Similar observations were found in the study conducted by Ramírez-Crescencio et al. and Mohraz $\mathrm{M}$ et al. ${ }^{7,8}$ Control of toxoplasmic infection needs cellular immunity, mediated by T-lymphocytes, macrophages, and activity of various inflammatory mediators such as type1 cytokines and interferon gamma. HIV-infected patients have impaired production of type 1 cytokines such as interleukin-2 and 12, and interferon gamma. This deficiency can enhance the development of toxoplasmosis infections in $\mathrm{HIV}$-positive patients. ${ }^{9,10}$

Lesions of tubercular etiology were the second most diagnosis observed. There is a high risk for developing tuberculosis in patients with HIV infection, as the immune response is insufficient to limit the growth of mycobacteria. Moreover, cell-mediated immunity suppression due to HIV infection inhibits the intracerebral immune response to mycobacteria. ${ }^{11}$ Tuberculosis of the CNS can be considered as the initial presentation of AIDS and potentially lifethreatening. Characteristic imaging findings from the radiologist is essential for the accurate diagnosis of the CNS tuberculosis, as it may resemble other infectious and noninfectious neurological conditions. ${ }^{12}$

In the current study, the majority of the lesions were observed in both supratentorial and infratentorial regions (58.8\%). Lesions can be in multiple locations of the brain, and this difference may reflect in the signs and symptoms of the patient. Altered mental status (94.1\%) and cognitive impairment (91.1\%) were the most prevalent symptom observed in the study group. HIV-associated systemic illness, accompanying immune dysfunction and complicated drug therapy may take part in the altered mental status. ${ }^{13}$ Similar to current observations of the study, studies conducted by Saini et al. and Chan et al. also found a significant level of neurocognitive impairment in $\mathrm{HIV}$-infected patients. ${ }^{14,15}$ Moreover, observations from Guevara-Silva, Sacktor et al. and Cross et al. showed significant improvement in cognitive impairment after antiretroviral therapy in HIVinfected patients. ${ }^{16-18}$ Functional alterations of the neurons by immunological and inflammatory changes due to HIVinfection may have role in the cognitive impairment. ${ }^{19-21}$

Majority of the lesions observed in T1-weighted MRI was hypointense $(70.6 \%)$. The reason behind this observation can be the high prevalence of toxoplasmosis in our study. Because there are various studies and case reports that showed hypointense lesions on T1-weighted MRI in toxoplasmosis. ${ }^{22-24}$ However, in T2-weighted MRI, a high prevalence of hyperintense lesions was observed (79.4\%). This also can be linked to the increased prevalence of toxoplasmosis in the study group, as T2-weighted MRI in toxoplasmosis shows hyperintensive lesions. Similar hyperintensive observations were found in various studies on toxoplasmosis. ${ }^{25,26}$

In immunocompromised patients, the lesions in toxoplasmosis found as central with petechial hemorrhage encircled by a ring of free tachyzoites, encysted bradyzoites, and inflammatory cells. ${ }^{27}$ Similar ring enhancement of the lesions was observed in the current study, particularly in toxoplasmosis (20.6\%) and tubercular etiology (17.6\%) on contrast T1-FASTAT sequence. A study conducted by Mahato et al. also found the significant role of tuberculosis 
as the leading cause of ring-enhancing lesions in Indian set up. ${ }^{28}$ Meningeal enhancement of lesions was absent in most of our patients (76.5\%). Tubercular etiology (8.8\%) was the major cause for the leptomeningeal enhancement of the lesions. Cases of toxoplasmosis, tubercular etiology, and lymphoma showed diffusion restriction on DWI sequence. This may be due to increased intracellular proportions in the pathological processes of those conditions.

\section{CONCLUSION}

MRI is useful to differentiate and characterize various brain lesions in HIV-infected patients. It is a noninvasive, radiation-free tool with multiplanar capabilities. Also, it has a high sensitivity in identifying lesions of the central nervous system. It is very beneficial, together with the results of other tests, in the final diagnosis of the lesions in the CNS. This plays an important role in guiding accurate diagnosis, treatment planning and monitoring the efficacy of the therapy in HIV-infected patients.

\section{REFERENCES}

1. Atluri VS, Hidalgo M, Samikkannu T, et al. Effect of human immunodeficiency virus on blood-brain barrier integrity and function: an update. Front Cell Neurosci. 2015;9(1):212.

2. Hazleton JE, Berman JW, Eugenin EA. Novel mechanisms of central nervous system damage in HIV infection. HIV AIDS (Auckl). 2010;2(5):39-49.

3. Le LT, Spudich SS. HIV-Associated Neurologic Disorders and Central Nervous System Opportunistic Infections in HIV. Semin Neurol. 2016;36(4):373-81.

4. Kranick SM, Nath A. Neurologic complications of HIV-1 infection and its treatment in the era of antiretroviral therapy. Continuum. 2012;18(6 Infectious Disease):1319-37.

5. Tate DF, Khedraki R, McCaffrey D, et al. The role of medical imaging in defining CNS abnormalities associated with HIV-infection and opportunistic infections. Neurotherapeutics. 2011;8(1):103-16.

6. Zhao F, Sun L-q, Tian Y-m, et al. The diagnostic value of brain magnetic resonance imaging in detecting CNS diseases among advanced AIDS patients. Infection International. 2014;3(4):173-8.

7. Ramirez-Crescencio MA, Velasquez-Perez L, RamirezCrescencio MA, et al. Epidemiology and trend of neurological diseases associated to HIV/AIDS. Experience of Mexican patients 1995-2009. Clin Neurol Neurosurg. 2013;115(8):1322-5.

8. Mohraz M, Jozani ZB, Behtaj M, et al. Neurological manifestations in HIV positive patients in Tehran, Iran. Asian Pac J Trop Dis. 2014;4(4):S481-S5.

9. Subauste CS, Wessendarp M, Portilllo J-AC, et al. Pathogen-specific induction of CD154 is impaired in CD4+ $\mathrm{T}$ cells from human immunodeficiency virusinfected patients. J Infect Dis. 2004;189(1):61-70.

10. Basavaraju A. Toxoplasmosis in HIV infection: An overview. Trop Parasitol. 2016;6(2):129-35.

11. Nelson CA, Zunt JR. Tuberculosis of the central nervous system in immunocompromised patients: HIV infection and solid organ transplant recipients. Clin Infect Dis. 2011;53(9):915-26.
12. Sanei Taheri M, Karimi MA, Haghighatkhah $\mathrm{H}$, et al. Central nervous system tuberculosis: an imagingfocused review of a reemerging disease. Radiol Res Pract. 2015;2015.

13. Ho EL, Jay CA. Altered mental status in HIV-infected patients. Emerg Med Clin North Am. 2010;28(2):31123.

14. Chan LG, Kandiah N, Chua A. HIV-associated neurocognitive disorders (HAND) in a South Asian population-contextual application of the 2007 criteria. BMJ Open. 2012;2(1):e000662.

15. Saini S, Barar KV. Assessment of neurocognitive functions in HIV/AIDS patients on HAART using the international HIV dementia scale. Int J Nutr Pharmacol Neurol Dis. 2014;4(4):252.

16. Guevara-Silva E. Cognitive impairment and antiretroviral treatment in a Peruvian population of patients with human immunodeficiency virus. Neurología (English Edition). 2014;29(4):224-9.

17. Sacktor N, Nakasujja N, Skolasky R, et al. Antiretroviral therapy improves cognitive impairment in HIV+ individuals in sub-Saharan Africa. Neurology. 2006;67(2):311-4.

18. Cross HM, Combrinck MI, Joska JA. HIV-associated neurocognitive disorders: antiretroviral regimen, central nervous system penetration effectiveness, and cognitive outcomes. S Afr Med J. 2013;103(10):758-62.

19. Gannon $\mathrm{P}$, Khan MZ, Kolson DL. Current understanding of $\mathrm{HIV}$-associated neurocognitive disorders pathogenesis. Curr Opin Neurol. 2011;24(3):275.

20. Saylor D, Dickens AM, Sacktor N, et al.HIV-associated neurocognitive disorder-pathogenesis and prospects for treatment. Nat Rev Neurol. 2016;12(4):234.

21. Sanmarti M, Ibáñez L, Huertas S, et al. HIV-associated neurocognitive disorders. J Mol Psychiatry. 2014;2(1):2 .

22. Macías N, editor Brain toxoplasmosis: typical and atypical imaging features2011: European Congress of Radiology 2011.

23. Ramachandran R, Radhan P, Anand R, et al. CNS toxoplasmosis in an immunocompetent individual. J Radiol Case Rep. 2014;9(1):908.

24. Smith AB, Smirniotopoulos JG, Rushing EJ. Central nervous system infections associated with human immunodeficiency virus infection: radiologic-pathologic correlation. Radiographics. 2008;28(7):2033-58.

25. Batra A, Tripathi R, Gorthi S. Magnetic resonance evaluation of cerebral toxoplasmosis in patients with the acquired immunodeficiency syndrome. Acta Radiol. 2004;45(2):212-21.

26. Lee GT,Antelo F, Mlikotic AA. Cerebral toxoplasmosis. Radiographics. 2009;29(4):1200-5.

27. Chong-Han $\mathrm{CH}$, Cortez SC, Tung GA. Diffusionweighted MRI of cerebral toxoplasma abscess. Am J Roentgenol. 2003;181(6):1711-4.

28. PS M, AS D, PB. T. Clinical and investigative profile of ring-enhancing lesions on neuroimaging. Indian J Clin Pract. 2012;22(4):512-8.

Source of Support: Nil; Conflict of Interest: None

Submitted: 12-11-2018; Accepted: 20-05-2019; Published online: 19-07-2019 